\title{
Post-transplant lymphoproliferative disorder of the cauda equina in a kidney transplant recipient
}

\author{
Yuichiro Nishiyama ${ }^{1} \cdot$ Akio Iwanami $^{1,2} \cdot$ Shuji Mikami ${ }^{3}$ - Soya Kawabata ${ }^{1}$ Osahiko Tsuji ${ }^{1}$ Narihito Nagoshi ${ }^{1}$. \\ Eijiro Okada ${ }^{1}$. Nobuyuki Fujita ${ }^{1}$ Mitsuru Yagi ${ }^{1}$ Ken Ishii ${ }^{1,4} \cdot$ Morio Matsumoto ${ }^{1}$ - Masaya Nakamura ${ }^{1}$. \\ Kota Watanabe ${ }^{1}$
}

Received: 10 August 2018 / Revised: 11 October 2018 / Accepted: 22 October 2018

(C) International Spinal Cord Society 2018

\begin{abstract}
Introduction Post-transplant lymphoproliferative disorder (PTLD) is a condition associated with post-transplant immunosuppression that can develop in various organs, including the grafted one. However, it has rarely been reported in nerve tissue. We encountered an unexpected case of PTLD in the cauda equina of a kidney transplant recipient who was being treated with chronic immunosuppressive therapies.

Case presentation The patient was a 39-year-old woman in whom lower limb muscle weakness appeared and progressed rapidly 10 years after kidney transplantation for glomerulonephritis. Magnetic resonance imaging (MRI) findings were suggestive of an intradural extramedullary tumor. Diagnosis of PTLD was established on open biopsy. Culprit immunosuppressants (tacrolimus, mycophenolate mofetil, and prednisolone) were discontinued, and rituximab and radiation therapy were started. The paraplegia gradually improved after drug discontinuation, and the lesion diminished in size 3 months after this series of treatment, and finally disappeared on MRI as of 1 year after treatment.

Discussion PTLD in the cauda equina is extremely rare, and only one case involving the cauda equina has been reported previously. Biopsy should be performed initially for definitive diagnosis, after which the suspected culprit immunosuppressants should be immediately discontinued.
\end{abstract}

\section{Introduction}

Post-transplant lymphoproliferative disorder (PTLD) is a condition that is associated with post-transplant immunosuppression. It shows some similarities with classic lymphomas but occurs in only immunosuppressed individuals post-transplantation. It can develop in various organs,

$\triangle$ Akio Iwanami

akioiwanami@gmail.com

1 Department of Orthopedic Surgery, Keio University School of Medicine, Tokyo, Japan

2 Department of Physiology, Keio University School of Medicine, Tokyo, Japan

3 Department of Diagnostic Pathology, Keio University School of Medicine, Tokyo, Japan

4 Department of Orthopedic Surgery, School of Medicine, International University of Health and Welfare, Narita-shi, Chibaken, Japan including the grafted organ, but its onset in nerve tissue is relatively rare. We recently encountered an extremely rare case of PTLD developing in the cauda equina in a kidney transplant recipient. Herein, we present this case with a review of the literature.

\section{Case presentation}

This 39-year-old woman presented with a chief complaint of muscle weakness in the right leg. At the age of 28 , she had undergone living-donor kidney transplantation from her father for end-stage renal disease caused by chronic glomerulonephritis. Since then, she had been on an oral immunosuppressant regimen consisting of tacrolimus $2.5 \mathrm{mg} /$ day, mycophenolate mofetil (MMF) $1000 \mathrm{mg} /$ day, and prednisolone $5 \mathrm{mg} /$ day. Ten years after transplantation, the patient began to experience right lower back pain and muscle weakness of the right leg. She consulted a neighborhood hospital, where magnetic resonance imaging (MRI) revealed no evident abnormality. One month later, 
the right leg muscle weakness intensified and she was admitted to another hospital. From the MRI findings, an intradural extramedullary tumor at the L2 level was suspected. She was then referred to our hospital.

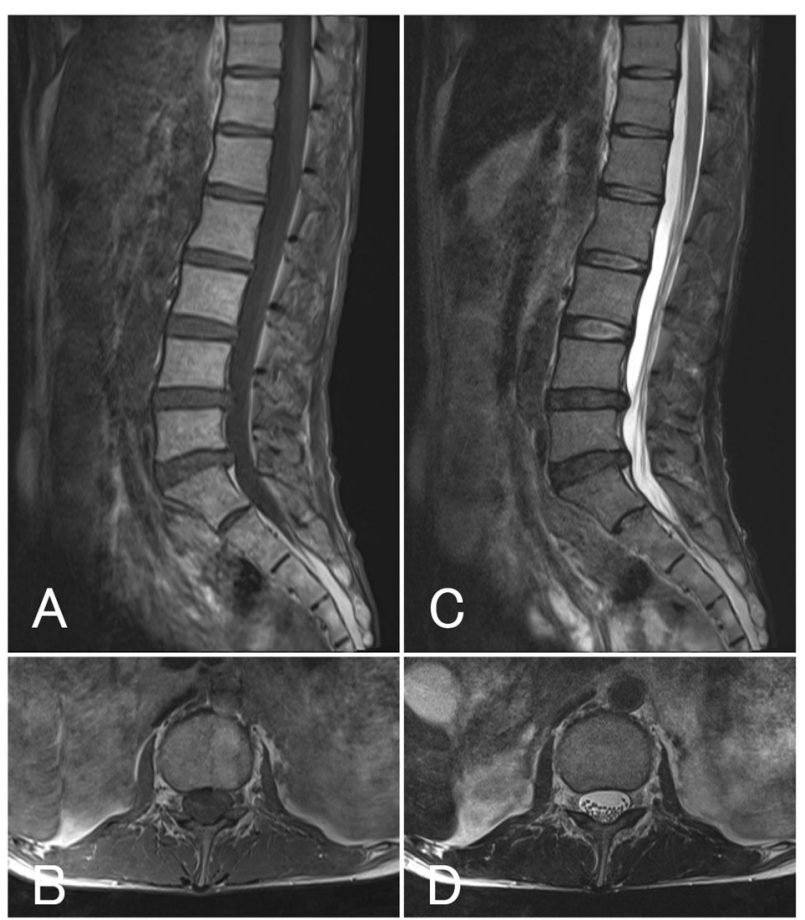

Fig. 1 Magnetic resonance imaging (MRI) scans at the time of symptom onset. a Sagittal T1-weighted image. b Axial T1-weighted image at the L2 vertebral body. c Sagittal T2-weighted image. d Axial T2-weighted image at the L2 vertebral body. No lesion is evident
Physical examination on admission revealed lower extremity paresis (involving the iliopsoas, quadriceps, anterior tibialis, gastrocnemius, peroneal, extensor hallucis longus, and flexor hallucis longus) with right dominance; a manual muscle test (MMT) score of 0 for the entire right leg muscle strength, an MMT score of 4 for the entire left leg muscle strength, and absent patellar and Achilles tendon reflexes in the right leg were observed. Reduced sensation was found at the L2 level and below on the right side, so she could neither walk nor stand. Dysuria was also noted, but bowel dysfunction was not observed. The Japanese Orthopaedic Association (JOA) score for lumbar spine disease was $(3,1,0)(2,0,0)(1,0,1,0,2,1,0)(-3)=8 / 29$, indicating severe physical impairment (the lower the score, the more severe the impairment). The JOA score evaluates lumbar spine disease using subjective symptoms, objective findings, daily living behavior, and bladder function [1].

Although no abnormal lesion was detected on initial MRI (Fig. 1), a repeat MRI 1 month later revealed a lesion (isointensity on T1-weighted imaging, low signal intensity on T2-weighted imaging, and uniform enhancement with gadolinium) in the intradural extramedullary area at the L2 level on the right side (Fig. 2). PTLD, hematoma, and metastasis were suspected based on the above findings and the lesion was biopsied for definitive diagnosis. After the dura and arachnoid mater were incised under microscopic guidance, the lesion was carefully observed. The cauda equina was inflamed and swollen, but there was no evidence of a tumor (Fig. 3). The inflamed tissue was resected for pathological examination. On histopathology, cluster of differentiation (CD)-20-positive and EBV-encoded small
Fig. 2 MRI scans 1 month after symptom onset. a Sagittal T1weighted image. b Sagittal T2weighted image. c Axial T2weighted image at the L2 vertebral body. d Sagittal T1weighted image with gadolinium enhancement. e Axial T1weighted image with gadolinium enhancement at the L2 vertebral body. A lesion (with isointensity on T1-weighted imaging and low signal intensity on T2weighted imaging with gadolinium enhancement) is visible on the right side inside the dura mater at the L2 level (arrows)
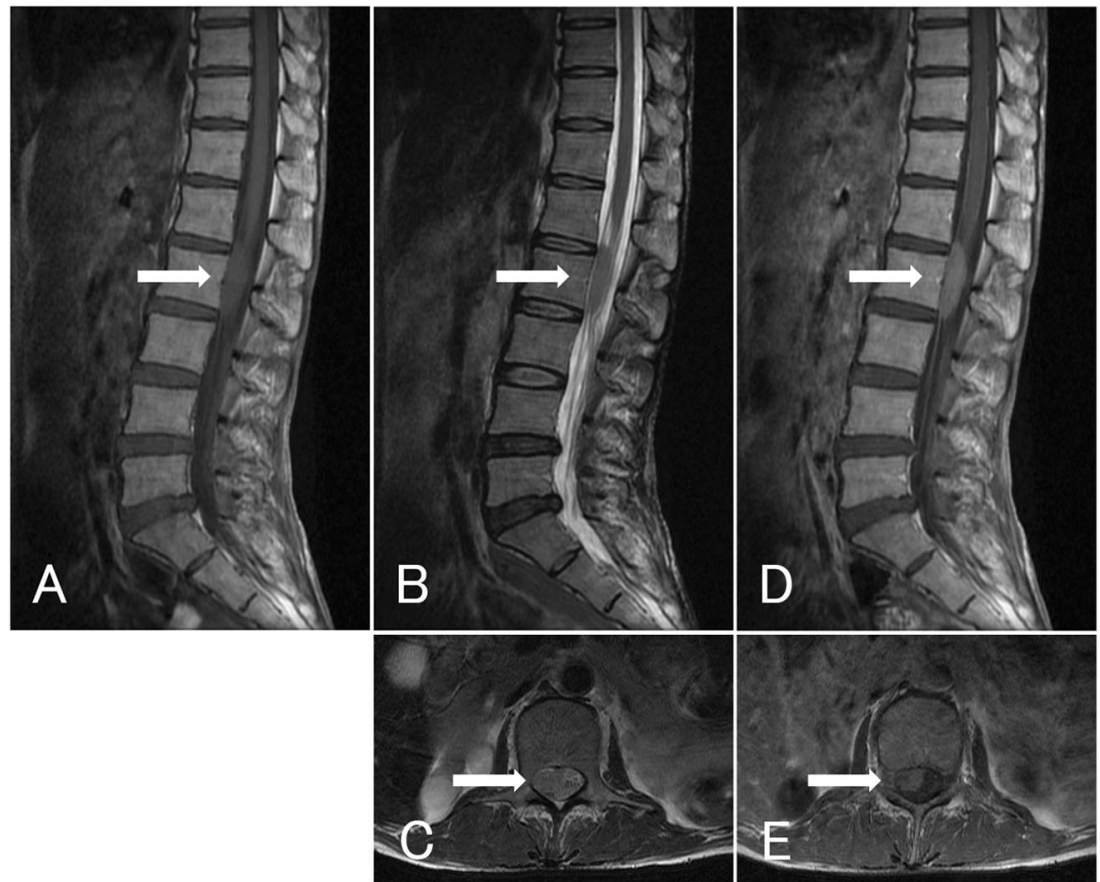


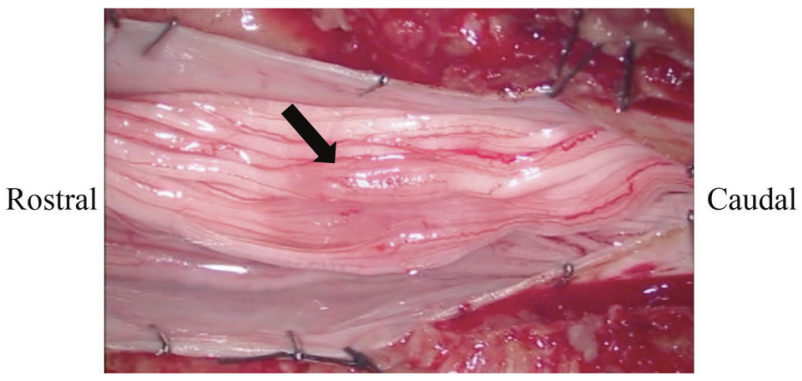

Fig. 3 Intraoperative findings. The cauda equina is swollen and inflamed (arrow). No tumorous lesion is evident
RNA (EBER)-positive B-cells with nuclear atypia were detected (Fig. 4). Blood tests demonstrated the presence of the Epstein-Barr virus (EBV) at $\leq 200$ copies/ $\mu \mathrm{g}$, but EBV viral capsid antigen immunoglobulin $G$ titer was 4.4 EIA (positive), EBV viral capsid antigen immunoglobulin $\mathrm{M}$ titer was 0.1 EIA (negative), and EBV virus nuclear antigen immunoglobulin $\mathrm{G}$ titer was 3.8 EIA (positive). Serum soluble interleukin-2 receptor level was elevated at $778 \mathrm{U} /$ $\mathrm{mL}$. Cerebrospinal fluid analysis revealed an elevated EBV copy number $(50,000$ copies $/ \mu \mathrm{g})$ with elevated cell count $(109 / \mu \mathrm{L})$ and total protein levels $(472 \mathrm{mg} / \mathrm{dL})$. At this point,
Fig. 4 Histopathological findings. a Hematoxylin-eosin (HE) staining, $\times 200$. b HE staining, $\times 400$. Large atypical lymphocytes with nuclear atypia are seen (black arrows). Normalsized lymphocytes without nuclear atypia are also seen (red arrows). c Anti-CD20 immunostaining, 200. d AntiCD20 immunostaining, $\times 400$. There is a positive chromatic response in the cytoplasm of relatively large-sized B cells (black arrow). e Anti-CD3 immunostaining, $\times 400$. f AntiCD3 immunostaining, $\times 400$. There is a positive chromatic response in the cytoplasm of small $\mathrm{T}$ cells (black arrow). There is no sign of nuclear atypia. g EBV-encoded small RNA (EBER) in situ hybridization, $\times 200$. h EBER in situ hybridization, $\times 400$. Epstein-Barr virus (EBV) is detected in the nuclei of large $B$ cells (black arrows). Polyclonal hyperplasia of EBV-positive Bcells is also appreciated

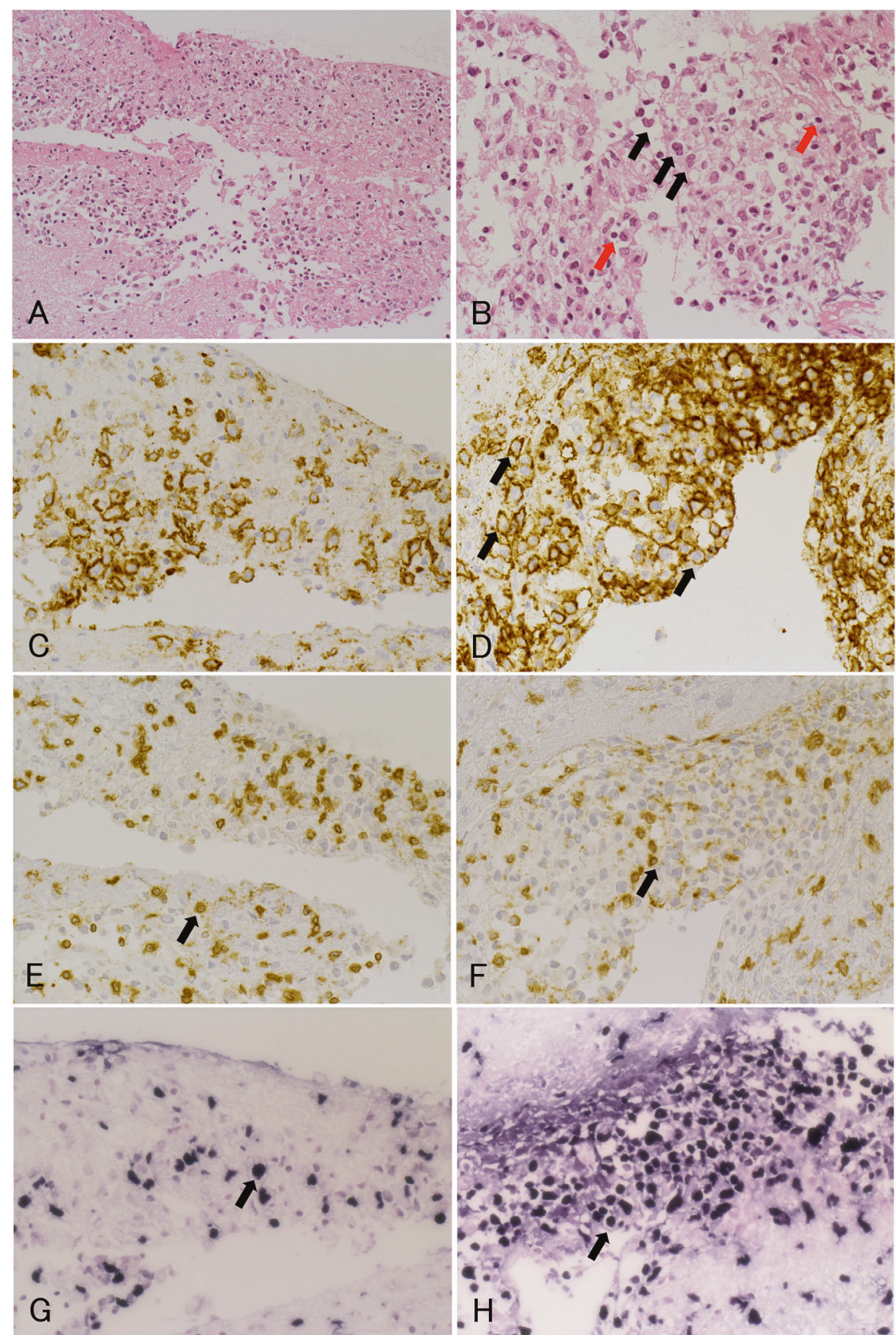



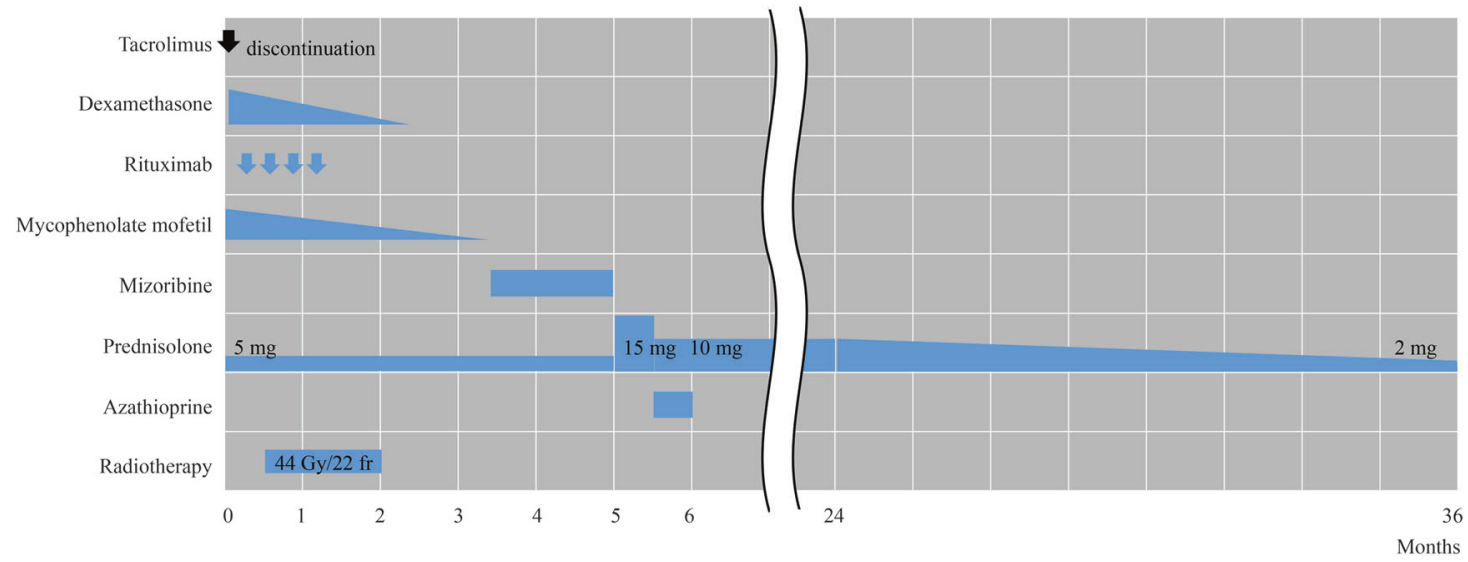

Fig. 5 Summary of the postoperative treatment. The horizontal axis shows the time course and the vertical axis shows treatment contents. Black arrow indicates discontinuation. Blue indicates the medicine or

we made a diagnosis of PTLD originating in the cauda equina after kidney transplantation.

No effective treatment has been established for PTLD. Since PTLD is caused by immunosuppressants, discontinuation is usually standard with radiotherapy, chemotherapy, and rituximab as appropriate. In this case, after discussion with the transplant surgeons, tacrolimus was discontinued, and oral steroids were begun. Rituximab $500 \mathrm{mg}$ ( $375 \mathrm{mg} / \mathrm{m}^{2}$ once weekly for 4 weeks) and radiotherapy (44 Gy/22 fractions to T12-L4) were also administered. The MMF dose was gradually reduced, and immunosuppressive therapy comprising mizoribine and azathioprine was initiated, with dose adjustments as needed. Finally, oral prednisolone $2 \mathrm{mg} /$ day was added. Figure 5 summarizes the course of the combined postoperative therapy. Physical therapies such as muscle strengthening, standing balance, and gait exercises were intensively performed in parallel every day. Three months after surgery, lower extremity strength had not improved but gradually recovered 1 year post-surgery. Subsequently, 1.5 years after surgery, the right leg muscle strength (for the iliopsoas, quadriceps, anterior tibialis, gastrocnemius, peroneal, extensor hallucis longus, and flexor hallucis longus) was MMT 2 and the left leg muscle strength had been fully recovered. Four years after surgery, the right iliopsoas and quadriceps strength had recovered to MMT4- and 3+ respectively. The patient could walk with a cane and anklefoot orthosis and bladder function had returned to normal. No rejection occurred after immunosuppressants were discontinued and renal function was maintained. On MRI, the lesion was unchanged 2 weeks after surgery, but was much smaller at 3 months and had completely disappeared by 1 year postoperatively and thereafter. No recurrence was seen as of at 3 years after surgery (Fig. 6). The patient's JOA score had improved from 8 to 17 at the final follow-up. This treatment being used. Triangles indicate that the amount of drug is being reduced gradually

study was approved by the institutional review board of our institution, and informed consent was obtained from the study participant prior to this case report.

\section{Discussion}

First reported in 1968, PTLD is a disease entity that is frequently encountered after solid organ or hematopoietic stem cell transplantation [2]. It shows some similarities with classic lymphomas. PTLD mostly originates from B cells, and EBV involvement has been suggested in many cases [3]. The incidence of systemic PTLD is approximately $20 \%$ [4-7]. It is common in patients after multipleorgan transplantation, such as bowel and heart-lung [6, 8]. On the other hand, the incidence of PTLD originating in the nervous system, including the central nervous system (CNS), is relatively small. PTLD originating in the CNS was first reported [9] in 1970, and it has been observed in $7-15 \%$ of all PTLD cases [10-14]. It is common in patients after pancreas and kidney transplantation [13]. There are only three case reports of PTLD developing at the T5 level in the spinal cord after kidney transplantation [15], one report of PTLD developing in the brain and cauda equina 1 year after hematopoietic stem cell transplantation [16] and one report of PTLD developing in the ventral aspect of the spinal cord 61 days after hematopoietic stem cell transplantation [17]. According to another report, PTLD developed in the spinal cord in $10 \%$ of 33 cases, but no further details were provided [12]. To the best of our knowledge, only one case of PTLD originating in the cauda equina has been reported previously [16] involving a patient with PTLD that developed in the cauda equina as well as the brain, which disseminated with a fatal outcome. 


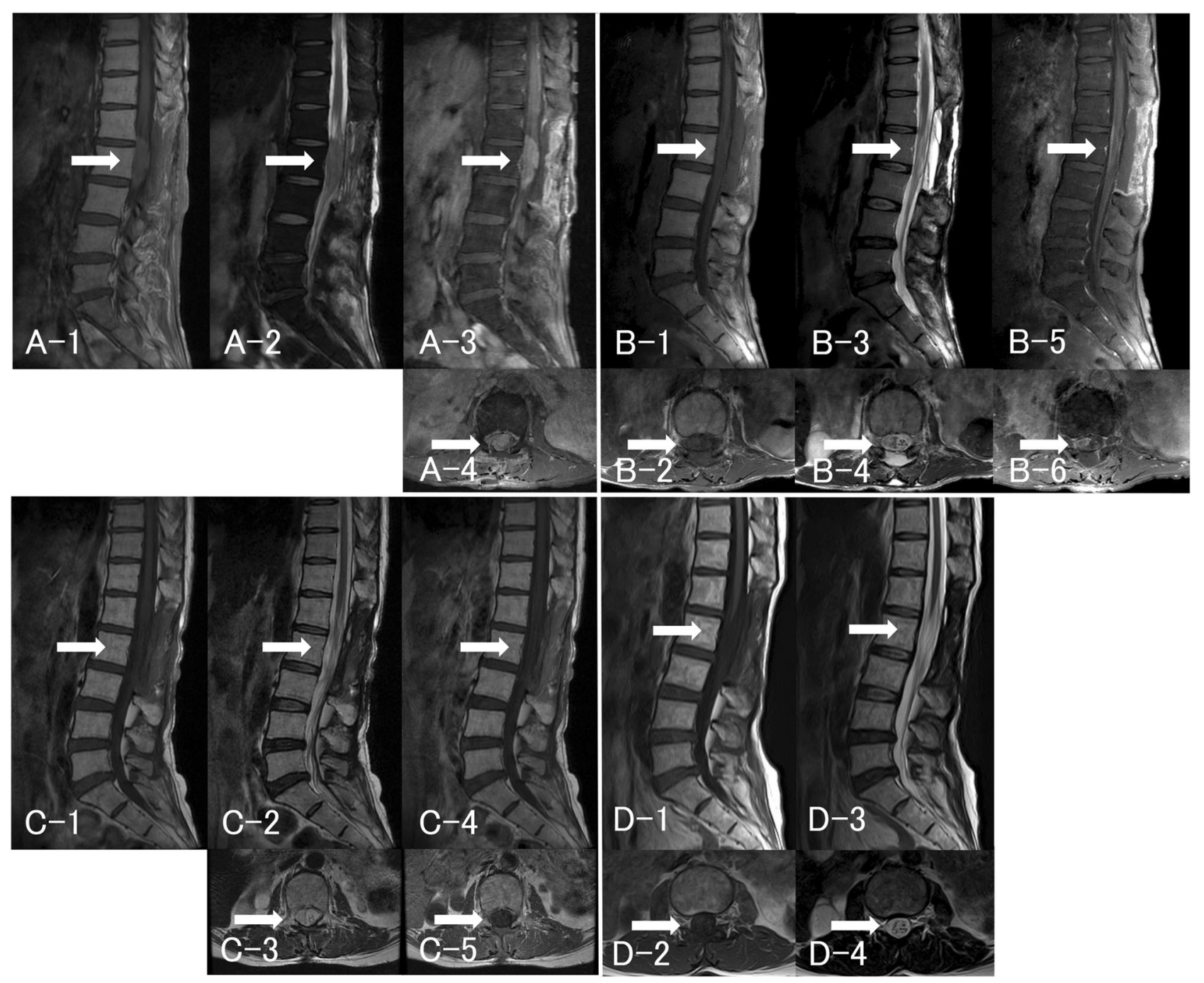

Fig. 6 a MRI scans 2 weeks after biopsy. (A-1) Sagittal T1-weighted image. (A-2) Sagittal T2-weighted image. (A-3) Sagittal T1-weighted image with gadolinium enhancement. (A-4) Axial T1-weighted image with gadolinium enhancement at the L2 vertebral body. The lesion is still recognizable (arrows). b MRI scans 3 months after biopsy. (B-1) Sagittal T1-weighted image. (B-2) Axial T1-weighted image at the L2 vertebral body. (B-3) Sagittal T2-weighted image. (B-4) Axial T2weighted image at the L2 vertebral body. (B-5) Sagittal T1-weighted image with gadolinium enhancement. (B-6) Axial T1-weighted image with gadolinium enhancement at the L2 vertebral body. The lesion has diminished in size in response to discontinuation of tacrolimus and

PTLD is usually diagnosed within a year after transplantation [4], while PTLD of the nervous system is diagnosed later, occurring about 12.6 to 54.0 months after transplantation in the literature [3,12,18-20]. CNS involvement has been shown to be a poor prognostic factor that is predictive of poor survival. On MRI, a frequently observed feature of PTLD of nervous system origin is uniform or ring-shaped enhancement. Histopathologically, the disease is monomorphic in most cases $[12,18]$. There are numerous reports on multiple PTLDs [3, 12, 19], and starting steroid and radiation therapy (arrows). c MRI scans 1 year after biopsy. (C-1) Sagittal T1-weighted image. (C-2) Sagittal T2weighted image. (C-3) Axial T2-weighted image at the L2 vertebral body. (C-4) Sagittal T1-weighted image with gadolinium enhancement. (C-5) Axial T1-weighted image with gadolinium enhancement at the L2 vertebral body. The lesion has completely disappeared on these images (arrows). d MRI scan 3 years after biopsy. (D-1) Sagittal T1-weighted image. (D-2) Axial T1-weighted image at the L2 vertebral body. (D-3) Sagittal T2-weighted image. (D-4) Axial T2weighted image at the L2 vertebral body. No recurrence is observed (arrows)

$35 \%$ of all cases with this type of PTLD present within 1 year after transplantation [3, 19].

Possible risk factors for onset of PTLD include use of cyclosporine and tacrolimus for immunosuppression and EBV infection in EBV-seronegative patients during immunosuppression [21, 22].

The plasma EBV-DNA level has also been considered useful for the diagnosis; a viral load exceeding $10^{4}$ copies/ $\mu \mathrm{g}$ DNA in peripheral blood is known to be diagnostically significant, and it has been reported that the EBV-DNA 
level in the cerebrospinal fluid is higher than that in the blood in some cases of PTLD [23, 24].

For primary treatment of PTLD, the culprit immunosuppressant therapy should be discontinued. However, long-term remission is difficult to achieve with this measure alone, and additional therapy may be necessary. Radiotherapy has been reported to extend patient survival [12, 19]. Methotrexate administration has also been reported to yield favorable outcomes [25, 26]. In CD20positive cases, the use of rituximab may be considered. Rituximab has been reported to be effective despite the prevailing view that the molecular weight of rituximab $(145 \mathrm{kDa})$ is too large to allow its passage through the blood-brain barrier [16, 27-31]. There is a report of improvement in progression-free survival in response to treatment with rituximab or cytarabine [18]; however, lower progression-free survival has been associated with low performance status and higher lactate dehydrogenase. The most significant prognostic factor reported is a lack of response to the initial treatment [18].

In the present case, PTLD developed in the cauda equina 10 years after transplantation. MRI revealed uniform contrast enhancement and higher cerebrospinal fluid EBV-DNA level than in blood, suggesting EBVassociated PTLD of nervous system origin. The lesion was biopsied to make a definitive diagnosis. Thereafter, the culprit immunosuppressant therapy was discontinued and replaced with rituximab (because the lesion was CD20-positive) followed by radiotherapy. Following treatment, the tumor disappeared on MRI and the patient's JOA score improved from 8 to 17 . This suspected case of PTLD was successfully controlled by discontinuation of the suspected culprit immunosuppressants and administration of rituximab, which has previously been reported to be an effective treatment for this disease, and radiotherapy [31].

Autonomic and sexual function is as important as muscle strength and bladder and bowel function after spinal cord injury and cauda equina failure [32, 33]. Although we cannot discuss them in this case since JOA score does not include autonomic and sexual function, we note that the Autonomic Standards Assessment Form of the American Spinal Injury Association/International Spinal Cord Society is useful for this purpose $[32,33]$.

We encountered a very rare case of PTLD developing in the cauda equina 10 years after transplantation. In managing a case of suspected PTLD, biopsy should be initially performed for definitive diagnosis. If PTLD is confirmed, the suspected culprit immunosuppressants should be discontinued immediately and the immunosuppressant regimen switched to rituximab therapy and radiotherapy if necessary.

\section{Compliance with ethical standards}

Conflict of interest The authors declare that they have no conflict of interest.

\section{References}

1. Izumida $\mathrm{S}$, Inoue $\mathrm{S}$. Assessment of treatment for low back pain. $\mathbf{J}$ Jpn Orthop Assoc. 1986;60:391-4.(in Japanese).

2. Doak PB, Montgomerie JZ, North JD, Smith F. Reticulum cell sarcoma after renal homotransplantation and azathioprine and prednisone therapy. BMJ. 1968;4:746-8.

3. Castellano-Sanchez AA, Li S, Qian J, Lagoo A, Weir E, Brat DJ. Primary central nervous system posttransplant lymphoproliferative disorders. Am J Clin Pathol. 2004;121:246-53.

4. Nagle SJ, Reshef R, Tsai DE. Posttransplant lymphoproliferative disorder in solid organ and hematopoietic stem cell transplantation. Clin Chest Med. 2017;38:771-83.

5. Opelz G, Dohler B. Lymphomas after solid organ transplantation: a collaborative transplant study report. Am J Transplant. 2004;4:222-30.

6. Cockfield SM. Identifying the patient at risk for post-transplant lymphoproliferative disorder. Transplant Infect Dis. 2001;3:70-8.

7. Opelz G, Henderson R. Incidence of non-Hodgkin lymphoma in kidney and heart transplant recipients. Lancet (Lond, Engl). 1993;342:1514-6.

8. DeStefano CB, Desai SH, Shenoy AG, Catlett JP. Management of post-transplant lymphoproliferative disorders. $\mathrm{Br} \mathrm{J}$ Haematol. 2018;182:330-43.

9. Schneck SA, Penn I. Cerebral neoplasms associated with renal transplantation. Arch Neurol. 1970;22:226-33.

10. Kempf C, Tinguely M, Rushing EJ. Posttransplant lymphoproliferative disorder of the central nervous system. Pathobiology. 2013;80:310-8.

11. Evens AM, David KA, Helenowski I, Nelson B, Kaufman D, Kircher SM, et al. Multicenter analysis of 80 solid organ transplantation recipients with post-transplantation lymphoproliferative disease: outcomes and prognostic factors in the modern era. J Clin Oncol. 2010;28:1038-46.

12. Cavaliere R, Petroni G, Lopes MB, Schiff D.International Primary Central Nervous System Lymphoma Collaborative G Primary central nervous system post-transplantation lymphoproliferative disorder: an International Primary Central Nervous System Lymphoma Collaborative Group Report. Cancer. 2010;116:863-70.

13. Buell JF, Gross TG, Hanaway MJ, Trofe J, Roy-Chaudhury P, First MR, et al. Posttransplant lymphoproliferative disorder: significance of central nervous system involvement. Transplant Proc. 2005;37:954-5.

14. Penn I, Porat G. Central nervous system lymphomas in organ allograft recipients. Transplantation. 1995;59:240-4.

15. Abdelhadi SM, Kondziella D, Kruse A, Pedersen M, ClasenLinde E, Larsen VA. [Post-transplant lymphoproliferative disorder is a rare cause of spinal cord compression]. Ugeskrift for laeger. 2015;177:2-3.

16. Traum AZ, Rodig NM, Pilichowska ME, Somers MJ. Central nervous system lymphoproliferative disorder in pediatric kidney transplant recipients. Pediatr Transplant. 2006;10:505-12.

17. de Beukelaar JW, van Arkel C, van den Bent MJ, van't Veer MB, van Doornum GJ, Cornelissen JJ, et al. Resolution of EBV $(+)$ CNS lymphoma with appearance of CSF EBV-specific T cells. Ann Neurol. 2005;58:788-92. 
18. Evens AM, Choquet S, Kroll-Desrosiers AR, Jagadeesh D, Smith SM, Morschhauser F, et al. Primary CNS posttransplant lymphoproliferative disease (PTLD): an international report of 84 cases in the modern era. Am J Transplant. 2013;13:1512-22.

19. Snanoudj R, Durrbach A, Leblond V, Caillard S, Hurault De Ligny B, Noel C, et al. Primary brain lymphomas after kidney transplantation: presentation and outcome. Transplantation. 2003;76:930-7.

20. Phan TG, O'Neill BP, Kurtin PJ. Posttransplant primary CNS lymphoma. Neuro Oncol. 2000;2:229-38.

21. Taylor AL, Marcus R, Bradley JA. Post-transplant lymphoproliferative disorders (PTLD) after solid organ transplantation. Crit Rev Oncol Hematol. 2005;56:155-67.

22. Lim WH, Russ GR, Coates PT. Review of Epstein-Barr virus and post-transplant lymphoproliferative disorder post-solid organ transplantation. Nephrol (Carlton, Vic). 2006;11:355-66.

23. Yaginuma $\mathrm{T}$, Yamamoto $\mathrm{H}$, Mitome J, Tanno $\mathrm{Y}$, Yamamoto I, Kobayashi A, et al. Successful treatment of monomorphic primary central nervous system post-transplantation lymphoproliferative disorder 5 years after kidney transplantation. Transplant Infect Dis. 2012;14:E102-6.

24. Hoshino Y, Kimura H, Tanaka N, Tsuge I, Kudo K, Horibe K, et al. Prospective monitoring of the Epstein-Barr virus DNA by a real-time quantitative polymerase chain reaction after allogenic stem cell transplantation. Br J Haematol. 2001;115:105-11.

25. Taj MM, Messahel B, Mycroft J, Pritchard-Jones K, Baker A, Height S, et al. Efficacy and tolerability of high-dose methotrexate in central nervous system positive or relapsed lymphoproliferative disease following liver transplant in children. $\mathrm{Br} \mathrm{J}$ Haematol. 2008;140:191-6.
26. Nabors LB, Palmer CA, Julian BA, Przekwas AM, Kew CE. Isolated central nervous system posttransplant lymphoproliferative disorder treated with high-dose intravenous methotrexate. Am J Transplant. 2009;9:1243-8.

27. Shah GD, Yahalom J, Correa DD, Lai RK, Raizer JJ, Schiff D, et al. Combined immunochemotherapy with reduced whole-brain radiotherapy for newly diagnosed primary CNS lymphoma. J Clin Oncol. 2007;25:4730-5.

28. Ruhstaller TW, Amsler U, Cerny T. Rituximab: active treatment of central nervous system involvement by non-Hodgkin's lymphoma? Ann Oncol. 2000;11:374-5.

29. O'Brien SM, Kantarjian H, Thomas DA, Giles FJ, Freireich EJ, Cortes $\mathrm{J}$, et al. Rituximab dose-escalation trial in chronic lymphocytic leukemia. J Clin Oncol. 2001;19:2165-70.

30. Gonzalez-Barca E, Domingo-Domenech E, Capote FJ, GomezCodina J, Salar A, Bailen A, et al. Prospective phase II trial of extended treatment with rituximab in patients with B-cell post-transplant lymphoproliferative disease. Haematologica. 2007;92:1489-94.

31. Choquet S, Leblond V, Herbrecht R, Socie G, Stoppa AM, Vandenberghe P, et al. Efficacy and safety of rituximab in B-cell post-transplantation lymphoproliferative disorders: results of a prospective multicenter phase 2 study. Blood. 2006;107:3053-7.

32. Courtois F, Alexander M, McLain ABJ. Women's sexual health and reproductive function after SCI. Top Spinal Cord Inj Rehabil. 2017;23:20-30.

33. Krassioukov A, Biering-Sorensen CF, Donovan W, Kennelly M, Kirshblum S, Krogh K, et al. International Standards to document remaining Autonomic Function after Spinal Cord Injury (ISAFSCI), First Edition 2012. Top Spinal Cord Inj Rehabil. 2012;18:282-96. 